\title{
PSYCHOLOGICAL CONSEQUENCES OF CORONAVIRUS: SUGGESTED SOLUTIONS FOR ATHLETES
}

\author{
Leila Heidaryani', Mohsen Keramatimoghadam², Majid Keramatimoghadam ${ }^{3}$ \\ ${ }^{1}$ PhD student in Educational Psychology, Lorestan University, Lorestan, Iran \\ ${ }^{2}$ Master of Cultural Studies and Media, Islamic Azad University, Central Tehran Branch, Tehran, Iran \\ ${ }^{3}$ PhD Student in Sports Management, Islamic Azad University, Boroujerd Branch, Boroujerd, Iran
}

Original scientific paper

\begin{abstract}
Coronaviruses are a large family of viruses and a subset of coronaviruses that range from the common cold virus to the cause of more serious illnesses such as SARS, Mers, and Covid. So far, however, seven human-transmitted coronaviruses have been discovered, the latest of which, the new coronavirus, spread to humans in December 2019 in Wuhan, China. It is observed that coronavirus disease due to unknown psychological consequences such as anxiety, stress, self-illness, obsessive-compulsive disorder, coronary phobia and finally depression and social isolation, which in addition to physical and physical injuries, causes serious psychological damage. Provided for members of the community; By focusing on the idea of positivity (resilience, hope, optimism, self-efficacy) and strengthening selfmanagement behaviors, as well as managing negative emotions such as fear, stress, anxiety, sadness, anger and depression to strengthen the immune system and We can go to war with the Corona virus and be sure to defeat it. Applying this method (positivity and focusing on good angles) at all levels of prevention and treatment of patients with physical diseases, including coronary heart disease, seems necessary.
\end{abstract}

Keywords: Psychological consequences, Coronavirus, Suggested solutions

\section{Introduction}

fear, stress, anxiety, sadness, anger, and depression, we can strengthen the immune system and fight the coronavirus and be sure to defeat it. Accordingly, considering that stress and anxiety are among the most common psychological consequences of coronavirus disease, which make the course of this disease worse; One of the suggested methods to deal with stress and anxiety is positive thinking (2) As a new branch of psychology, it pays attention to the scientific study of human strengths and happiness. In fact, by focusing on a positive outlook versus a negative outlook that is followed by issues such as stress, fear, anxiety, problems, and psychological illness; We can help improve and increase mental well-being, quality of life and happiness (3). Therefore, using this method at all levels of prevention and treatment of patients with physical diseases seems necessary (4). It is important to note here that not all people respond to the stressful situations of this disease in the same way, and the emotional impact of this critical situation on the individual can affect the personality traits and experiences of the individual, environmental, economic and social conditions. Depending on the individual and the community, as well as the level of access to support resources. For example, children and adolescents' reactions to
Coronaviruses are a large family of viruses and a subset coronaviruses that range from the common cold virus to the cause of more serious illnesses such as SARS, Mers, and Covid. Coronaviruses were discovered in 1965 and continued to be studied until the mid-1980s. The virus is naturally prevalent in mammals and birds, yet seven human-transmitted coronaviruses have been discovered so far. The latest of these, the new corona, broke out in December 2019 in Wuhan, China, with a human pandemic (1). Corona disease, due to its unknownness, creates a situation of fear and anxiety in the body, which in addition to physical and bodily injuries, inflicts a psychological shock on the individual and society. This anxiety and stress, in addition to sometimes causing certain and unusual behaviors by some people, It is also an important factor in making it harder to deal with the threat of the virus, including indifference to preventive measures. Although it seems natural to react anxiously to difficult situations, what is very important here is, Prolonged duration of anxiety, which weakens the immune system and increases the chances of people getting the disease. Therefore, we must deal with the part of anxiety that is under our control and reduce it by doing various activities such as exercise, study, learning skills, watching movies, and so on. By managing negative emotions such as 
severe anxiety can have adverse health effects. Just as excessive anxiety can cause a person significant problems and risks, the absence of anxiety is not without its drawbacks. Mild anxiety can be constructive and force a person to try to do things in life in a timely and sufficient manner (6).

Self-morbidity: Self-morbidity is a type of mental disorder that is characterized by a person's belief in a serious illness and concern about it. Selfmorbidity is caused by misinterpretation of physical symptoms and related information; The main feature of self-morbidity is a clinical picture whose prominent disorder is the unrealistic interpretation of physical symptoms and natural sensations as abnormal symptoms that lead to mental occupation or the belief in fear of a serious illness (7). Four theories have been proposed for the etiology of self-morbidity disorder. According to the first theory, people may focus on and misinterpret bodily feelings and view them as a warning because of incorrect cognitive schema. In the second theory, based on the social learning model with emphasis on the two processes of observation and imitation, the presence of an infected relative in the family can be considered as a risk factor for self-morbidity disorder. The third theory holds that this disorder is another form of other mental disorders and is most commonly associated with depression and anxiety disorders. The fourth approach to self-morbidity disorder is the psychodynamic approach, according to which aggressive and hostile desires towards others turn into physical complaints (8).

Algebraic obsessive-compulsive disorder: The main manifestation of obsessive-compulsive disorder is mental occupation with order, perfectionism and individual mental control, which ends at the cost of loss of flexibility, foresight and interpersonal efficiency.

\section{Main features:}

Based on new research findings, three main and pivotal features can be identified in obsession:

Abnormal risk assessment

- Pathological suspicion

- Incompleteness

These key characteristics are manifested in behaviors such as excessive checking, washing, and the need for uniformity and order.

Obsessive-compulsive disorder is concerned with the occurrence of an event with a very low probability (9)

Corona phobia: The stress and anxiety caused by this disease has disrupted the lives of some people and deprived them of sleep and food, severe stress can be expressed in different ways, some react immediately to stress, and some may show traumatic signs and symptoms over time. Therefore, our role is to respond appropriately to the symptoms of their psychological s-ester, given the awareness and cognition we have of our children and those around us. Take the time to answer their questions, if their information and awareness of this disease is unrealistic and covered by their anxiety and fear, we try to replace it with realistic and correct information, because our ignorance and misinformation It can cause confusion and ultimately a source of stress and anxiety. In relation to children, parents play a very important role, keeping the parents calm and calm in this situation is very important, if parents seem too worried and anxious, the anxiety of children and other family members may increase. Parents need to strengthen their children's sense of security by reassuring them that they will not get sick if they take personal hygiene and preventative care. Therefore, they should use this period of quarantine as a good opportunity to interact directly with children and even thereby improve their individual and interpersonal skills. In this regard, the purpose of writing this article is a brief overview of the psychological consequences of coronavirus and to offer suggested solutions in this regard.

\section{Psychological consequences}

Anxiety: Anxiety is a state that a person experiences while waiting for a danger. Anxiety originates from a thought or an idea and then transmits to emotions and physical actions, resulting in increased heart rate, sweating and tension. These three mental, physical and behavioral factors can each affect the other. Anxiety causes unpleasant experiences. It is often difficult to describe such a state, but it can be discussed under two categories, one is what we feel in the brain and the other is the manifestations we see in our body. In our minds, intense knowledge of stress and unhappiness, fear of distress, and anticipation of unfortunate events cause anxiety, which causes human restlessness, so we must do something to either get rid of the source of anxiety or Escape from it; There is a feeling of anxiety in our body and a feeling of tension that, if intensified enough, causes us to feel sick, so the heart beats faster, the body trembles and we sweat more than usual (5). Anxiety disorders originate from a range of psychological factors. For example, a person who is in a special job and social position, responsibilities are assigned to him and people have higher expectations of him, he becomes anxious. The severity of anxiety varies from person to person. Mild anxiety is a level of normal excitement in people, but moderate to 
these people seem to tolerate problems better than others and recover later; We even have many Qur'anic verses in this regard that emphasize tolerance and patience in difficult situations. For example, in verse 45 of Surah AlBaqarah, it is stated that "and ask God for help in seeking patience in hardships and hardships" or in verse 127 of Surah An-Nahl, it is stated that "be patient for what has befallen you." Therefore, resilience can be suggested as a vital system for maintaining health and performance against stress and anxiety, and it is assumed that stubbornness and resilience prevent the harmful effects of stress. Dependent diseases protect against stress (12). Resilience is a skill to get through life's problems even when there are unfavorable and difficult conditions, such as tying a resilient rope around problems that helps a person pull them back when they are badly injured. Look into the eyes of men. Therefore, resilience as an ability in human beings causes them to successfully overcome and adapt to unfavorable conditions and achieve emotional and social ability despite facing life problems and critical situations (13). Here it is important to note that one of the important factors in creating this trait is resilience, having kind family ties and family cohesion. As long as the family environment is calm and the necessary coordination is established between the parents and they support their children well, the home environment will become more lovable for everyone and thus the conditions will be created for cultivating resilience. It is obvious that chaos, disorder, conflict and quarreling between parents, cause stress and anxiety in the family, especially in children, and the house becomes an unsuitable place. The second factor is altruism: the more one is in an altruistic environment and does altruistic work and strengthens one's altruistic spirit, the more resilient it becomes (14).

Hope: Hope is a state of positive motivation; People with high hopes have a great ability to create paths to achieve their goals. This psychological resource constantly creates the attitude that problems will be solved and goals will be achieved. In addition, people with high expectations frame their tasks in a way that keeps them highly motivated to succeed in the tasks they are performing (15). Schneider points out that when people face obstacles, thinking of agency or the power of will in the structure of hope becomes especially important (16). When faced with such obstacles, agency (goal-oriented energy) helps the individual to be motivated to find the best alternative.

Optimism: Optimism is having a positive view of expected results or creating positive citations which weakens the immune system and ultimately makes the body prone to this disease. We often see and hear that people are in a state of fear, while real horror is seen more in movies than in life. People live in stress $70 \%$ of the time, and living in stress means living for survival. All living things can withstand shortterm stress. Therefore, it should be noted that it is normal to have some stress and anxiety in the current situation, but if the stress response is activated and you cannot stop it, you are now prone to disease because no living thing can survive in this emergency for a long time. It is a scientific fact that stress hormones disrupt the order of genes and cause disease. Because of the size of the neocortex in their brains, humans can activate stressful responses just by thinking about their problems, which means that our thoughts can both make us sick and make us feel better.

\section{Other consequences}

In addition to causing fear, stress, anxiety and other obsessive behaviors in the society in the current situation, Corona virus, but if we do not manage and control our mental health, it is possible that this disease will have long-term effects and consequences. It will be more destructive. For example, due to the fact that the culture of communication in Iranian society is more physical and face-to-face, but in the current situation, the sudden cessation of this communication, may over time provide conditions for depression and social isolation for individuals. On the other hand, considering that every day we see repeated recommendations about regular hand washing and disinfection hygiene regarding not getting coronary heart disease, these cases, if followed in a very extreme way, can lead to obsessive-compulsive disorder. Provides a person with serious effects that are not less than the coronary heart disease itself; However, we must also be aware of this and hope that with very good mental health we can avoid coronary heart disease.

\section{Proposed psychological strategies for proper treatment of coronary heart disease:}

Resilience: Resilience means having proper performance in a hazardous tissue; In other words, a person's successful coping with stressors and difficult situations is called resilience (10). Resilient people have the power to accept reality and believe that life is more meaningful than wanting to succumb to adversity (11). Philosophers and social scientists, after years of research, found many people who adapted to unpleasant life situations; In popular culture, there are people who are known for their traits such as "resilient" and "patient", 
1. Exposure to successful experiences by setting achievable goals and thereby increasing the likelihood of performance.

2. By exposing the person to appropriate role models who are successful in practice, thereby adding to his or her successful succession experiences.

3- There are different ways to provide, persuade and verbal encouragement for successful performance; For example, I can say that I have the ability to function successfully. I can successfully overcome this crisis and problem.

4. Strength and vital energy can be increased by boosting physiological arousal through diet and exercise programs.

5 - Searching for the unknown, welcoming change and accepting that the beauty of life is in its change.

6- Feeling of belonging, lack of self-esteem and self-satisfaction towards one's fellow human beings.

7 - Mobility and dynamism in the face of injustice and discovering a creative solution and maintaining peace.

8- Paying attention to self-motivation and internal motivating forces.

9- Not having a glorious life and not complaining; With a low sense of self-efficacy, one shares one's glories with others to gain their support.

10- Learning useful tips from problematic situations as much as learning from success conditions.

Bandura has successfully used these techniques to increase self-efficacy in situations such as overcoming stress and anxiety and physical pain and healing disease (21).

\section{Self-management behaviors:}

Self-management is the process by which individuals persuade, guide, and guide themselves in order to achieve a particular behavior, function, or goal, or in the process of achieving them; This structure has a broad structure that includes three complementary behavioral strategies (self-awareness, management of core behaviors, self-monitoring, self-motivation, self-accountability, self-reward, and personal goal setting (natural reward strategy). Positive experiences related to performing tasks, creating a pleasant, enjoyable and useful image of the current situation (constructive thought model strategy) and having positive and constructive thought patterns against destructive patterns in dealing with problems and turning them into opportunities for people in Achieving high levels of self-management helps (22). The benefits of having high levels of self-management in patients include improving the quality of life, expanding levels of self-confidence, improving about events, and optimists expect positive and desired results. Optimists cite external, specific, and unstable factors when they have a bad experience. Scarwild sees the pessimist as someone who knows the price of everything but knows the value of nothing. Because overoptimism can lead to optimism or unrealistic goals, sometimes having a flexible and realistic optimism can be more beneficial. Optimism is associated with increased motivation, success, greater physical health, and mental well-being (17).

Efficacy: Everyone faces problems and obstacles along the way. The way human beings deal with these obstacles and problems goes back to their personality traits. People's beliefs organize their world and give meaning to their experiences. How these beliefs can create a psychological world in people and lead them to different thoughts, feelings, and actions in similar situations is a very important issue. Self-efficacy is defined as an individual's confidence to perform a behavior, which is essential for overcoming problems and achieving desired goals. Self-efficacy is also an important prerequisite for successful self-management and behavior change. According to Bandura theory, self-efficacy includes a person's confidence and confidence in the ability to perform self-care tasks optimally, so that the person achieves the desired results. Studies show that people with high scores on self-efficacy focus on problems and tasks and show more effort, but people with low scores become anxious about problems and activities and struggle to find solutions. They become difficult (18). According to Bandura and Luke, self-efficacy, with increased perseverance and hard work, causes people to experience emotional adjustment, higher success and higher health (19). Therefore, self-efficacy is an excellent mechanism for promoting health and the results of studies have shown that selfefficacy regulation is effective in modulating health-related behaviors such as anxiety and stress in disease conditions. Improving selfefficacy leads to better self-management outcomes, increases life expectancy, and modulates health behaviors. Improving selfefficacy is associated with symptom control, treatment, physical outcomes, and psychosocial effects in chronic patients (20). For this reason, a sense of self-efficacy enables people to do extraordinary things using their skills in dealing with obstacles. Effective performance requires both having the skills and believing in the ability to perform those skills. . Managing constantly changing, ambiguous, unpredictable and stressful situations requires multiple skills. Strategies for overcoming weaknesses and achieving self-efficacy and success: 
strengths and happiness. In fact, by focusing on a positive outlook versus a negative outlook that is followed by issues such as stress, fear, anxiety, problems, and psychological illness; We can help improve and enhance mental well-being, quality of life and happiness.

In this situation, resilience, patience and perseverance are the right skills to get through the difficulties and endure the unfavorable and difficult conditions that currently exist. On the other hand, hoping for the end of the problems and leaving behind the critical situation today, as well as hoping for the medical staff of the Ministry of Health to discover the vaccine for this disease, as a positive motivation in this situation will be very useful and constructive. We should also be optimistic that good news is on the way and the situation will improve day by day and positive things will happen in connection with this coronary situation. And finally, with selfefficacy and the belief that we can get through difficult situations, and by joining selfmanagement behaviors, we will be able to get through these critical situations and get back on track to a normal life without a corona. Therefore, using this method (positivity and focusing on good angles) at all levels of prevention and treatment of patients with physical diseases, including coronary heart disease, seems necessary. their inner views and emotions about themselves and the environment, and ultimately improving mental health.

\section{Conclusion}

According to this article, the occurrence of various psychological consequences such as anxiety, self-morbidity, obsessive-compulsive disorder, coronary phobia, depression, obsessivecompulsive disorder and social isolation due to coronary heart disease is not unexpected. In this situation, when we cultivate negative thoughts in the mind, they are repeated over and over again in the mind, creating stronger negative thoughts. That is why we lose a significant part of our abilities without realizing it. The symptoms of this loss of abilities are not only obvious in health and psyche, but also this disability manifests itself in the human body, turning a person into a weak person and leading him to various diseases, including disease. Will provide a corona. These toxins are the result of negative weaving, which can also destroy the body by creating negative emotions such as stress, anger, anxiety, fear and health. But in the meantime, focusing on positivity can be considered a very practical method of coping, which as a new branch of psychology pays attention to the scientific study of human

\section{REFERENCES}

1. Tyrrell, D.A. (1965-06-05). cultivation of a novel type of common- cold virus in organ cultures. British medical journal. $1(\Delta \leftarrow \varphi \wedge), 1 \% q V-V$.

2. Ahangarzadeh Rezaei, S; Ouladrostam, N \& Nemat Elahi, A. (2017). The effect of positive thinking training on stress, anxiety and depression in patients with coronary heart disease. Journal of Urmia School of Nursing and Midwifery, 15 (5), 448 -439.

3. Seligman, M. (2003). Learned Theory of Helplessness and Positive Psychology. Translation, Karimi, Ramin, Tehran: Danjeh. (2010).

4. Ghadmpour, E; Heidariani, L \& Radmehr, F. (2019). The effectiveness of group therapy based on acceptance and commitment on cognitive flexibility and life satisfaction of women with multiple sclerosis. Journal of Health Psychology, 8 (1), 153- 167.

5. Asadi, R. (2016). Determining the effect of positive treatment on anxiety of mothers with children with bipolar disorder. Master Thesis, Arak Azad University.

6. Motlagh, M \& Asadi, Z. (2009). The effect of cognitive behavioral therapy in reducing anxiety in adolescent girls with generalized anxiety disorder. Journal of Research in Exceptional Children, 9 (1), 25- 34.

7. Deale A. (2008). Psychopathology and treatment of severe health anxiety. Anxiety Discord, 22, 240-46.

8. Talaei, A; Fayyazi Bordbar, M- R; Nasiraei, A; Samari, A- A; Mirshjaeian, M \& Rezaei Ardani, A. (2009). Self-morbidity and its related factors in students of Mashhad University of Medical Sciences. Journal of Ilam University of Medical Sciences, 17 (1), 8-19.

9. Asgari, K. (2008). Obsessive-compulsive disorder - a combination of theoretical and practical dimensions. Thought and Behavior, 3 (3), 61-72.

10. Klivand Thani, R. (2018). The effectiveness of positive psychology on students' resilience, selfefficacy and test anxiety. Master Thesis, Qom University.

11. Rahimi, S. (2015). The effectiveness of positive psychology interventions on the resilience of female patients with multiple sclerosis. Specialized database of Noor magazines. 
12. Majidian, F. (2007). Investigating the Relationship between Self-Efficacy Beliefs and Hardiness with Job Stress in High School Principals in Sanandaj. Master Thesis, Allameh Tabatabai University of Tehran.

13. Inzlich M, Aronson J, Good C \& McKay L. (2006). A particular resiliency to threatening environments. Journal of Experiment Tall Social Psychology, 4(2), 323- 336.

14. Sarami, M. (2015). The effect of positive group therapy on the psychological well-being of children of divorce. Master Thesis, Kharazmi University.

15. Snyder, C. R. (2000). Handbook of hope. San Diego: Academic Press.

16. Snyder, C. R. (2002). Hope theory: Rainbows in the mind. Psychological Inquiry, 13, 249- 276.

17. Yousefvand, L \& Radmehr, P. (2018). Investigating the relationship between psychological capital and organizational climate with job motivation (case study of Khorramabad). Research project report, Lorestan Disciplinary Command Research Office.

18. Bandura, A. (1986). Social 3333/ foundations of thought and action. A social cognitive theory. Englewood, Cuffs, NJ Hall. Inc.

19. Bandura, A. Locke, E.A. (2003). Negative self- efficacy and goal effects revisited. Journal of portal Apple Psychology. 88(1), 87- 99.

20. Baljani, E; Rahimi, J; Salimi, S \& Aggressive, M. (2011). The effect of nursing interventions in promoting self-efficacy and reducing vascular risk factors in cardiovascular patients. Journal of Nursing and Midwifery, Tehran University of Medical Sciences, 17 (1), 45- 54.

21. Gholami, M- T. (2009). Investigating the Relationship between Collective Effectiveness Beliefs and Self-Efficacy Beliefs with JobSatisfaction of Male Teachers. Master Thesis, University of Tehran.

22. Mirsafian, H- R; Afshari, M \& Kalateh Seifri, M. (2018). The effect of applying cybernetic management model on the development of self-management behaviors and its role on the development of psychological empowerment of employees and model presentation. New Approaches in Sports Management, 6 (21), 35-50.

\section{Correspondence to:}

Majid Keramatimoghadam

PhD student, Boroujerd University

E-mail: majid.keramatimoghadam@gmail.com 